\title{
Endoscopic ultrasound-guided transesophageal thoracentesis
}

In a retrospective, single-center study, we identified nine consecutive patients (six male; median age 66 years; range 55-79 years) who, between January 2003 and April 2007, underwent attempted endoscopic ultrasound (EUS-)guided thoracentesis into the right $(n=7)$ or left $(n=2)$ pleural space. In all patients a diagnosis of cancer was made either prior to $(n=6)$ or by $(n=3)$ EUS. Thoracentesis was not the primary indication for EUS in any patient. Right-sided thoracentesis was performed when possible due to the dependent position of the pleural fluid relative to the right esophageal wall in the left lateral decubitus position. Prior to aspiration, no additional maneuvers (i.e. patient breath hold) were performed. Thoracentesis was successful in all patients, and a median of $12 \mathrm{~mL}$ (range $2.5-36 \mathrm{~mL}$ ) was aspirated without complications. Pleural fluid cytology was positive for malignancy in two patients ( $22 \%)$ : adenocarcinoma of unknown primary $(n=1)$ and metastatic ovarian adenocarcinoma $(n=1)$. In both cases, EUSguided thoracentesis provided the initial diagnosis of a malignant effusion ( Fig. 1-4). Pleural fluid cytology in each of the remaining seven was benign.

Following EUS, all patients were given one dose of an intravenous antibiotic (ampicillin/sulbactam or ciprofloxacin), and a prescription for an additional 3-5 days of oral antibiotic treatment (amoxicillin/clavulinic acid or ciprofloxacin). Follow-up chest radiographic imaging (median 3 months; range 1-18 months) in the seven with benign cytology showed complete or near complete resolution of all effusions.

Traditional thoracentesis using a percutaneous posterior approach without image guidance may be associated with pneumothoraces in approximately $10 \%$ of patients [1]. With sonographic guidance, the rate of pneumothorax following diagnostic percutaneous thoracentesis remains $2.5 \%-5.5 \%[2,3]$. There are limited data on the utility of EUS-guided thoracentesis $[4,5]$. Our series shows that EUSguided thoracentesis is technically feasible and safe, and may provide the initial diagnosis of a malignant pleural effusion
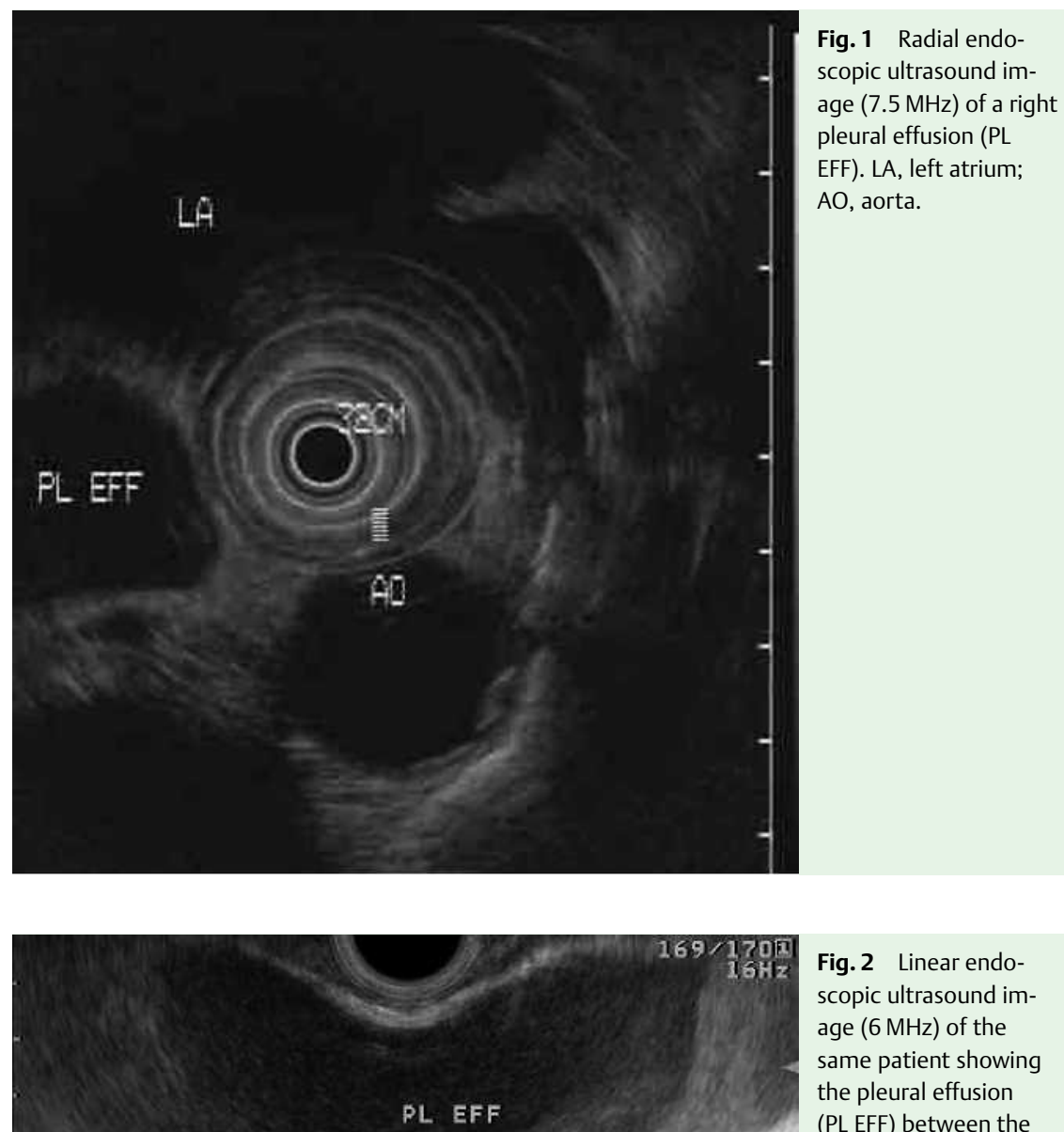

Fig. 2 Linear endoscopic ultrasound image $(6 \mathrm{MHz})$ of the same patient showing the pleural effusion (PL EFF) between the esophageal wall and the right lung. The pleura is demonstrated as the hyperechoic line between the pleural fluid and the lung. 


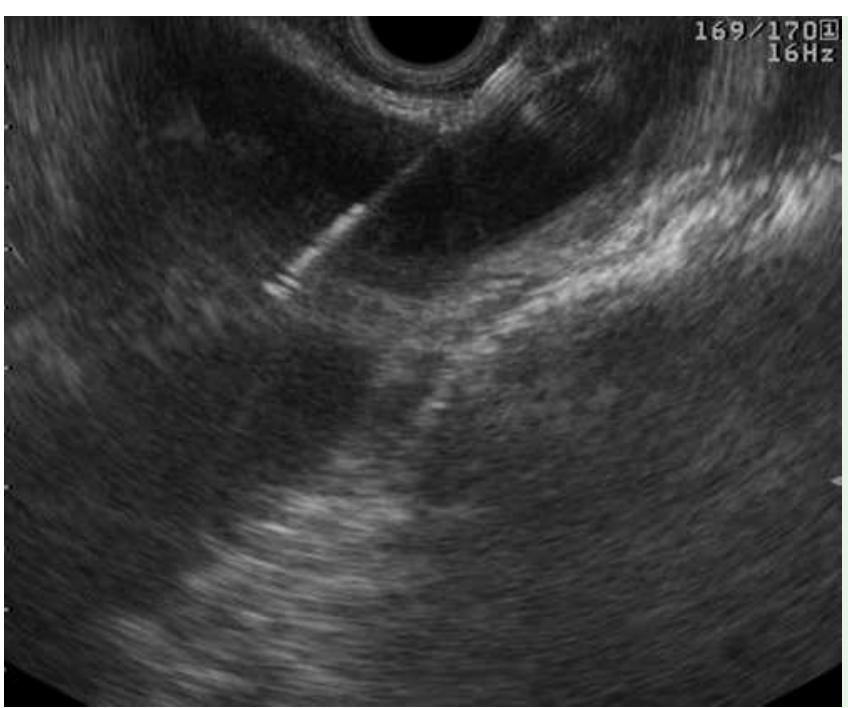

Fig. 3 Endoscopic ultrasound-guided fine needle aspiration of the pleural effusion. The tip of the needle is seen within the pleural cavity.

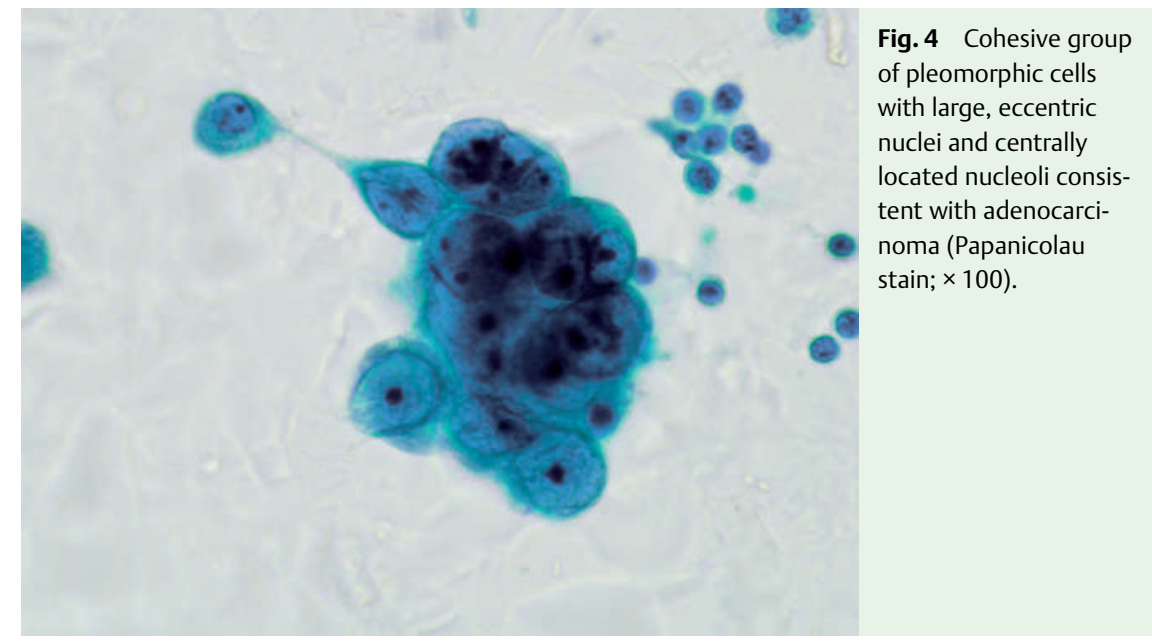

J. DeWitt ${ }^{1}$, P. Kongkam ${ }^{1}$, S. Attasaranya $^{1}$, J. K. LeBlanc ${ }^{1}$, S. Sherman ${ }^{1}$, F. D. Sheski ${ }^{2}$

1 Division of Gastroenterology and Hepatology, Department of Internal Medicine, Indiana University Medical Center, Indianapolis, Indiana, USA

2 Division of Pulmonary and Critical Care Medicine, Department of Internal Medicine, Indiana University Medical Center, Indianapolis, Indiana, USA
Bibliography

DOI 10.1055/s-2007-995399

Endoscopy 2007; 39: E118-E119

(c) Georg Thieme Verlag KG Stuttgart · New York . ISSN 0013-726X

\section{Corresponding author}

\section{J. M. DeWitt, MD}

Department of Medicine

Division of Gastroenterology Indiana University Medical Center 550 N. University Blvd., UH 4100 Indianapolis

IN 46202-5121

USA

Fax: +1-317-278-8144

jodewitt@iupui.edu 